\title{
An experimental study of the interface pressure profile during level walking of a new suspension system for lower limb amputees
}

\author{
Arezoo Eshraghi a, Noor Azuan Abu Osman a, Hossein Gholizadeh a, Sadeeq Ali a, \\ Stefán Karl Sævarsson b, Wan Abu Bakar Wan Abas a
}

a Department of Biomedical Engineering, Faculty of Engineering, University of Malaya, 50603 Kuala Lumpur, Malaysia

b Department of Biomedical Engineering, University of Calgary, Canada

Introduction

Suspension systems are necessary components of lower limb

prostheses and they are used to create a secure coupling between

the residual and prosthetic limbs. The majority of contemporary suspension

systems utilize silicone liners as the preferred suspension

system (McCurdie et al., 1997). Lower limb amputees have stated preference towards these silicone liners as a result of the fact that

these systems provide a close match to the residual limb, superior

suspension, improved appearance and better function (Baars and

Geertzen, 2005). In general, there is a high overall satisfaction with prosthetic devices that incorporate silicone liners as suspension systems (Eshraghi et al., 2012a). There are a variety of silicone suspensions in use that are coupled to the hard socket either by a distal single pin or through circumferential seal or seals that produce vacuum at the socket wall. Prosthetic hard sockets that are used with silicone suspension should be undersized to ensure a total-surface bearing fit. Research has revealed that a total-surface bearing socket exposes the soft tissue to tolerable compression (Laing et al., 2011). On the other hand, bony structures are stabilized within the residual limb; therefore the skin may not be damaged due to unbearable excessive pressure when these liners are in use (Wlodarczyk, 2007). Moreover, total-surface bearing sockets coupled with enhanced vacuum (for instance by Seal-In liners) might control volume fluctuation and perspiration. At the same time, piston motion or displacement within the socket and thereby shear force will be reduced.

Some researchers have attempted to evaluate the load applied to the residual limb either through completion of clinical assessments that use different types of transducers (Convery and Buis, 1999; Laing et al., 2011; Polliack et al., 2000; Sanders, 1998; Zhang et al., 1998) or through simulation techniques (Commean et al., 1997; Lin et al., 2004; Silver-Thorn and Childress, 1996). Friction within the prosthetic socket has a two-fold effect as it helps to retain the prosthesis on the 
residuum but at the same time it may distort the soft tissue (Mak et al., 2001). If large friction occurs at an interface, stressmay be localized and this can lead to the deformation of the remaining tissue. Conversely, Zhang et al. found that lubricating the skinwill increase the interface pressure (Zhang et al., 1996). Few research studies have dealt with the effect of liners and prosthetic sockets on the pressure applied to the residual limb. Without understanding the changes imposed on the soft tissue and skin by different socket designs and suspension systems, it is not possible to evaluate the overall prosthetic fit. Moreover, prosthetic interface pressure is believed to be a determinant of the amputees' comfort (Dou et al., 2006; Jia et al., 2004; Sanders et al., 2006;

Sewell et al., 2000).

Research has shown that pin liners exert compression on the residual limb proximally and tension distally during the swing phase of gait. This skin stretch at the pin site is called milking. This milking phenomenon is probably the cause of the short (edema and redness) and long-term (discoloration and thickening) transformations that are observed, particularly at the distal end of the residuum (Beil and Street, 2004). This compression can result in pain, discomfort and residual limb atrophy or volume loss.

A new prosthetic suspension system has been developed by the authors (Eshraghi et al., in press). This study aimed to compare the effect of this new prosthetic suspension system with pin/lock and Seal-In systems with regard to the interface pressure that is produced between the liner and socket. The researchers hypothesized that the new suspension system would result in less traction at the distal end of the residual limb and lower compression proximally in comparison to the pin/lock liner. The researchers also assumed that the Seal-In liner would offer similar interface pressure to the new suspension system, particularly at the distal region of the stump.

Methods

Fifteen amputees agreed to participate in the study as sample of convenience and were asked to sign a written consent form. Ethical approval was obtained from the University of Malaya Ethics Committee prior to the study. The subjects were required to conform to the following criteria in order to be included in the study: no ulcer on the residuum, no volume change at the residual limb and the ability to ambulate without assistance. As the minimum length for eligibility to use Seal-In liners is $11 \mathrm{~cm}$ based on the manufacturer guidelines, only those amputees with adequate residuum length were eligible to participate. The subjects were also considered for participation if they had used prosthesis in the last 6 months.

Each subject was provided with three new prostheses, each of 
which incorporated a different suspension system: (a) the new magnetic suspension system, (b) a pin/lock liner and (c) a suction Seal-In suspension. All the procedures from the casting to prosthetic alignment were performed separately for each prosthesis by one of the researchers (a registered prosthetist). All the prostheses incorporated a Flex-Foot Talux. All the experiments were carried out in the Motion Analysis Laboratory at the University of Malaya, while the subjects walked on the level ground wearing each of the three prostheses.

The new suspension system comprised (a) amounting plate coupled to the distal end of the silicone liner and (b) a magnet assembly embedded in the distal end of the hard socket (Fig. 1). The plate was a cup-shaped metal part that had a diameter thatmatched that of the distal liner. A screw through the middle of the plate connected the plate to the liner. The plate was filled with the silicone adhesive all around the central screw (Eshraghi et al., in press). A mechanical switch knob enabled the attachment to and detachment from the liner and the hard socket. When the switch knob was rotated, a magnetic field was produced and rotation in the opposite direction weakened the magnetic field so that the suspension failed (the liner was detached from the socket)

Dermo® liner (Össur, Reykjavik, Iceland) was used with both of the new suspension systems and the pin/lock suspension. The suction Seal-In system was a Seal-In® X5 liner (Össur, Reykjavik, Iceland) and an expulsion valve was mounted on the hard socket (Fig. 1).

In order to check the interface pressure, four F-socket transducers 9811E (Tekscan Inc., South Boston, USA) were employed. It is generally accepted that the sensors used to measure for interface pressure should be as thin as possible (Kim et al., 2003). The paper-thin F-socket sensors had a thickness of $0.18 \mathrm{~mm}$, good flexibility and high resolution. The sensor mats were trimmed according to the residuum counters and were located on the anterior (Ant), posterior (Pos), medial (Med) and lateral (Lat) surfaces of the residuum. In order to avoid displacement 


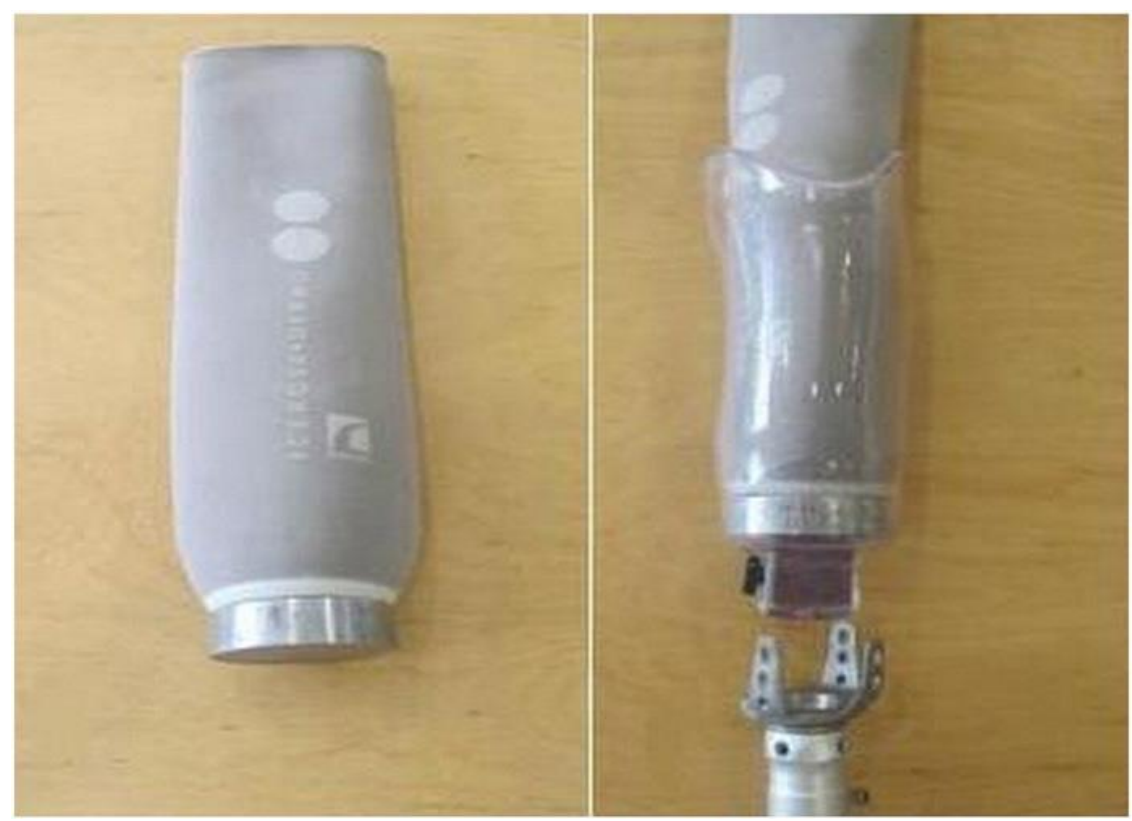

Fig. 1. New magnetic suspension system.

adhesive spray (3M Spray Mount Adhesive, 3M corporate, St. Paul, USA) was employed to secure the sensormats to the residual limb before the silicone liners were rolled on the transducers (Fig. 2).

Prior to the experiments, the transducers were calibrated to eliminate variation between each load cell. Following the manufacturer's instructions, two processes of equilibration and calibration were performed.

The sensorswere inserted individually into a pressure bladder connected to an air compressor and a constant pressure of $100 \mathrm{kPa}(20 \mathrm{psi})$ was applied for equilibration. Next, the calibration was accomplished according to each subject's body weight

In order to identify the gait cycle, force plate data was simultaneously gathered alongside the pressure data using two Kistler force plates at $50 \mathrm{~Hz}$. The subjects were asked to walk at a self-selected speed on a 10-meterwalkway. Prior to the data collection, the participants practiced the procedure. The frequency of data acquisition was $50 \mathrm{~Hz}$. The subjects completed five trials on the walkway. The average of the middle steps (excluding the two first and the two last) for the five trials was chosen for the analyses.

The assumption of normality and homogeneity of variance was verified using the Kolmogorov-Smirnov test. Afterwards, the differences in peak pressure values were definedwithin four transducer sites (anterior, posterior, medial, lateral) and suspension systems using a $4 \times 3$ (sensor $\times$ suspension systems) repeated measure analysis of variance (ANOVA). If the ANOVA showed significant differences, paired-samples t tests were used to compare mean peak pressures in different regions of the socket 
among the three suspension systems. Each sensor was further divided into three sub-regions, namely, proximal, middle and distal. All the statistical analyses were accomplished using SPSS 20.0 (SPSS, Chicago, IL, USA).

\section{Results}

Out of 15 subjects, three subjects were withdrawn from the study as they failed to complete the fitting and gait training sessions. The demographic data of the remaining 12 subjects is depicted in Table 1.

The analyses of data for four sensor arrays (three regions for each) were performed for the three suspension systems. First, the data was normalized to 100 percent of gait cycle. Repeated measure analysis of variance showed significant differences between the studied systems in some of the sensor sites during one gait cycle. Table 2 represents the average peak pressure values and the significant differences observed. There were also significant differences evident between the four sensor sites for each system. In the case of the magnetic lock, there was significant increase in the mean peak pressure at the anterior surface in comparison to the posterior, medial and lateral (79.26 vs. 26.01, 38.07, and 27.41 respectively). The same was true for the pin/lock and Seal-In systems (Table 2).

For the Seal-In liner, the mean peak pressures (APP) were higher in the proximal and middle of the sensor compared to the distal region at the anterior, posterior and medial surfaces of the residuum. Overall, the APP of the four sensor array sites during one gait cycle was higher for the Seal-In system compared to both the pin/lock liner and the new magnetic system.

The whole surface APP at the anterior aspect was lower with the magnetic system than it was with the pin/lock system during one gait cycle (79.26 vs. $89.89 \mathrm{kPa}, \mathrm{P}=0.034, \mathrm{t}=2.581)$. There was also increased APP with the pin/lock system at the posterior aspect of the residual limb during gait cycle (47.22 vs. $26.01 \mathrm{kPa}, \mathrm{P}=0.000$, $\mathrm{t}=9.254$ ). Comparative analysis of the pin/lock system to the new magnetic system revealed that there was no significant difference between the two during the stance. Nevertheless, significantly less mean peak pressures were seen with the new system during the swing phase of gait (Table 3).

Overall, the highest percentage of change was recorded for the posterior sensor between the new magnetic lock and the Seal-In system $(60.16 \%)$ and the least was between the pin/lock and new magnetic lock at the medial surface $(2.90 \%)$. When comparing the new magnetic lock with the pin/lock system, the percentage of change for all four sensor sites was more than $10 \%$, with the exception of the medial site. 
With regard to the distribution of pressure over the anterior surface,

the largest change was seen immediately after heel strike for

the pin/lock and Seal-In systems during one gait cycle. Conversely,

the largest change was observed at late stance with the new magnetic

system (Fig. 3). As for the posterior surface, a more homogenous

pattern was seen for all the suspension systems during gait, with

the greatest change at early stance (Fig. 3).

For all over stance, the average peak pressure at the distal region

of the anterior surface remained higher than the proximal portion

for all three suspension types (Fig. 4). The distal area of the posterior

surface demonstrated lower pressure than the proximal region. The

only exception was the Seal-In system, which produced higher

pressure at the middle region in comparison to the proximal area.

Full text is available at :

http://ac.els-cdn.com/S026800331200229X/1-s2.0-S026800331200229X-main.pdf?_tid=708db3c4-a018-11e3a5c2-00000aab0f6c\&acdnat $=1393551438$ 3b731019deaaea892a0ec74bf152801e

http://www.ncbi.nIm.nih.gov/pubmed/23157843

http://www.sciencedirect.com/science/article/pii/S026800331200229X 